TTR

Traduction, terminologie, rédaction

\title{
Le traducteur en coulisse : traduction de scénarios et productions audiovisuelles multilingues
}

\section{Hugo Vandal-Sirois}

Volume 27, numéro 2, 2e semestre 2014

Traduction, textes, médias

Translation, Texts, Media

URI : https://id.erudit.org/iderudit/1037745ar

DOI : https://doi.org/10.7202/1037745ar

Aller au sommaire du numéro

\section{Éditeur(s)}

Association canadienne de traductologie

ISSN

0835-8443 (imprimé)

1708-2188 (numérique)

Découvrir la revue

Citer cet article

Vandal-Sirois, H. (2014). Le traducteur en coulisse : traduction de scénarios et productions audiovisuelles multilingues. TTR, 27(2), 49-70.

https://doi.org/10.7202/1037745ar

\section{Résumé de l'article}

Les défis et enjeux de la traduction audiovisuelle, particulièrement ceux du doublage et du sous-titrage, ont été largement explorés en traductologie. L'analyse des contraintes liées à cette forme de traduction (l'interdépendance du visuel et du verbal, la synchronisation labiale et les restrictions temporelles ou spatiales, entre autres) a permis de cerner les mécanismes et les stratégies de la communication audiovisuelle multilingue. Toutefois, un volet de cette forme de traduction spécialisée demeure généralement méconnu, et ce, bien qu'il soit en plein essor et qu'il entraîne des difficultés traductionnelles uniques : la traduction de scénarios, dont les enjeux sont de taille. Plutôt que de fournir un document final (comme un film doublé ou sous-titré), le traducteur de scénarios doit, en plus de maîtriser le langage technique, l'élaboration de structures narratives et la rédaction de dialogues fluides et crédibles, produire un outil de travail sur lequel reposeront les activités des acteurs, du réalisateur et des techniciens. En cette époque marquée par la mondialisation et la multiplication des technologies de communication, et où les échanges culturels se déroulent sur la multitude d'écrans qui nous entourent, la traduction scénaristique représente un secteur d'activités aussi important que dynamique. Elle soulève des questions particulièrement intéressantes en traductologie, puisqu'elle place le traducteur au coeur du processus créatif, notamment dans le contexte de doubles tournages, au cours desquels le traducteur est parfois appelé à participer aux différentes étapes de production. Cet article propose de situer la traduction de scénarios dans le spectre plus large de la traduction audiovisuelle, d'en examiner les caractéristiques et défis, et, finalement, d'aborder le phénomène des doubles tournages, particulièrement dans le domaine de la publicité.
Ce document est protégé par la loi sur le droit d'auteur. L’utilisation des services d’Érudit (y compris la reproduction) est assujettie à sa politique d'utilisation que vous pouvez consulter en ligne.

https://apropos.erudit.org/fr/usagers/politique-dutilisation/ 


\title{
Le traducteur en coulisse : traduction de scénarios et productions audiovisuelles multilingues
}

\author{
Hugo Vandal-Sirois \\ Université de Montréal
}

\section{Résumé}

Les défis et enjeux de la traduction audiovisuelle, particulièrement ceux du doublage et du sous-titrage, ont été largement explorés en traductologie. L'analyse des contraintes liées à cette forme de traduction (l'interdépendance $\mathrm{du}$ visuel et du verbal, la synchronisation labiale et les restrictions temporelles ou spatiales, entre autres) a permis de cerner les mécanismes et les stratégies de la communication audiovisuelle multilingue. Toutefois, un volet de cette forme de traduction spécialisée demeure généralement méconnu, et ce, bien qu'il soit en plein essor et qu'il entraine des difficultés traductionnelles uniques: la traduction de scénarios, dont les enjeux sont de taille. Plutôt que de fournir un document final (comme un film doublé ou sous-titré), le traducteur de scénarios doit, en plus de maîtriser le langage technique, l'élaboration de structures narratives et la rédaction de dialogues fluides et crédibles, produire un outil de travail sur lequel reposeront les activités des acteurs, du réalisateur et des techniciens. En cette époque marquée par la mondialisation et la multiplication des technologies de communication, et où les échanges culturels se déroulent sur la multitude d'écrans qui nous entourent, la traduction scénaristique représente un secteur d'activités aussi important que dynamique. Elle soulève des questions particulièrement intéressantes en traductologie, puisqu'elle place le traducteur au cœur du processus créatif, notamment dans le contexte de doubles tournages, au cours desquels le traducteur est parfois appelé à participer aux différentes étapes de production. Cet article propose de situer la traduction de scénarios dans le spectre plus large de la traduction audiovisuelle, d'en examiner les caractéristiques et défis, et, finalement, d'aborder le phénomène des doubles tournages, particulièrement dans le domaine de la publicité.

Mots-clés: traduction audiovisuelle, scénario, double version, publicité, mondialisation 


\begin{abstract}
The challenges and issues of audiovisual translation, especially those of dubbing and subtitling, have been widely explored in translation studies. The analysis of the constraints in this field (the interdependence of visual and verbal elements, lip synchronization and temporal or spatial restrictions, among others) helped to better understand the mechanisms and strategies of multilingual audiovisual communication. However, an important part of this field of specialized translation remains largely ignored, although it is booming and implies unique translational difficulties: the translation of scripts. This activity raises interesting issues. Instead of providing a final document (as a dubbed or subtitled movie), the translator must, in addition to mastering the technical jargon, the development of narrative structures and the writing of fluid and believable dialogue, produce a useful work tool for the director, the actors and the technicians involved in the production. In addition, the translation of scripts is almost never mentioned in translation studies, even in this era of globalization and telecommunication technologies, where many cultural exchanges take place in the various screens that surround us and where it represents an important and dynamic sector of activity. This type of translation places the translator in the heart of the creative process, especially in the case of the double shoot, where he or she may even be involved in various stages of production. This article aims to locate the translation of scripts in the broader spectrum of audiovisual translation, to examine its characteristics and challenges, and to study the phenomenon of the double shoot, particularly in the field of advertising.
\end{abstract}

Keywords: audiovisual translation, script, double shoot, advertising, globalization

$\mathrm{Au}$ cours des dernières années, la traduction audiovisuelle s'est taillé une place de choix en traductologie et dans les programmes universitaires. Articles, numéros spéciaux de revues scientifiques, livres et colloques entièrement consacrés à cette forme de traduction sont aujourd'hui monnaie courante. Après avoir produit des publications générales d'ordre théorique ou pratique (v., p. ex., Orero, 2004; Díaz Cintas, 2009), consacrées principalement aux deux types de traduction audiovisuelle les plus fréquents que sont le doublage et le sous-titrage, et des études comparatives menées à partir de corpus formés en grande majorité de produits de divertissement, bien des chercheurs présentent désormais des études ciblées qui se concentrent sur un aspect, une contrainte ou un enjeu particuliers de la communication multimédia multilingue. On peut penser, par exemple, aux travaux sur des médias spécifiques comme la télévision ou les jeux vidéo (O’Hagan, 2009 et Pedersen, 2011, notamment) et à 
ceux qui portent sur des types de traduction audiovisuelle encore peu étudiés tels que le surtitrage ou la description sonore, ou encore à ceux qui s'attardent aux implications socioculturelles ou pédagogiques de la traduction audiovisuelle (comme The Didactics of Audiovisual Translation, Díaz Cintas, 2008). Au-delà de cette spécialisation normale et souhaitable des travaux de recherche, il importe de voir si les études traductologiques progressent en suivant l'évolution multimodale du texte audiovisuel et, surtout, si elles couvrent la totalité des facettes théoriques et pratiques de cette sphère d'activité.

Après un retour sur l'espace qu'occupe la traduction audiovisuelle en traductologie, nous nous proposons dans le présent article d'examiner un type de traduction audiovisuelle encore très peu exploré même s'il comporte des difficultés traductionnelles aussi uniques qu'intéressantes et qu'il permet d'aborder la question de la traduction d'un texte imbriqué dans un environnement de sons et d'images sous une perspective complètement différente: la traduction de scénarios. Nous nous pencherons également sur le rôle du traducteur dans la production de doubles versions, une pratique qui, bien qu'elle demeure dans l'ombre du doublage et du sous-titrage, est aussi courante en Europe qu'en Amérique du Nord. Nous conclurons sur l'analyse d'un exemple récent de publicité canadienne produite en double version.

\section{Recontextualisation de la traduction audiovisuelle}

La pratique de la traduction audiovisuelle comporte de nombreux défis, tant techniques que linguistiques et sémantiques, et soulève bien des questions, notamment quant aux conséquences du choix $\mathrm{du}$ mode de traduction audiovisuelle sur la réception du texte cible. Le traducteur qui se spécialise dans cette pratique doit considérer à la fois des informations verbales et non verbales, des messages exprimés ouvertement et d'autres, sous-entendus. $\mathrm{Ou}$ encore, il doit considérer comment le contenu sonore ou visuel d'une scène (une intonation choisie ou un geste posé par un personnage, par exemple) modifie le sens des paroles entendues par le spectateur. Ces préoccupations, qui combinent les dimensions linguistique et culturelle aux normes techniques et aux contraintes spatiotemporelles, sans oublier la dimension $\mathrm{du}$ respect des personnages et de la trame narrative dans le cas des œuvres de fiction, constituent hors de tout doute un terreau 
riche à explorer en traductologie. Toutefois, un autre facteur, d'ordre socioéconomique cette fois, peut expliquer l'intérêt sans cesse croissant pour la traduction audiovisuelle. En effet, alors que la mondialisation favorise les rapprochements politiques, économiques et commerciaux entre états et nations, le traducteur est davantage appelé à jouer les rôles clés de négociateur et d'agent culturel. L'actuelle hausse exponentielle des besoins de télécommunication est, bien entendu, rendue possible grâce au développement rapide des technologies de l'information, et l'écran semble être le lieu privilégié de ces rencontres multiculturelles. Les demandes et les besoins de traduction audiovisuelle progressent par conséquent, en raison notamment de la prolifération du contenu en ligne et des chaînes spécialisées de télévision, et la présence de la traduction audiovisuelle en traductologie évolue parallèlement à cette tendance.

\section{Létude du texte audiovisuel sous toutes ses formes... ou presque!}

Le texte audiovisuel se présente sous plusieurs formes qui ont toutes des caractéristiques et des normes bien distinctes, en plus de confronter le traducteur à des contraintes et défis variés. Les traductologues ont au fil du temps fait un survol assez complet des formes de traduction audiovisuelle, mais, il faut le dire, de manière bien peu équilibrée. En effet, la majorité des publications sur le sujet se concentrent sur la traduction des paroles de personnages et de narrateurs, au cinéma et à la télévision, par l'entremise du doublage et du sous-titrage. Ces travaux portent souvent sur les limites imposées au traducteur par le contenu visuel (mouvements des lèvres des acteurs dans le cas du doublage, désignation d'objets dans le cadre de la caméra ou hors champ, gags dont l'effet repose simultanément sur l'image et le son, etc.). D'autres études s'intéressent aux divergences entre le doublage et le soustitrage, du point de vue de la réception et de la compréhension du spectateur ou de celui des informations ayant été modifiées ou retranchées pour faciliter la lecture rapide d'un long monologue sous-titré, par exemple.

Il est facile de comprendre pourquoi le doublage et le sous-titrage, particulièrement ceux de grandes productions cinématographiques ou télévisuelles, inspirent autant les traductologues. Ces deux techniques imposent au traducteur 
de véritables casse-têtes sémantiques, culturels et techniques dont les solutions sont souvent fascinantes à observer. De plus, avec l'hégémonie états-unienne du divertissement culturel, elles constituent peut-être les formes de traduction auxquelles nous sommes le plus régulièrement exposés (et elles représentent par le fait même une industrie florissante). Par ailleurs, l'arrivée du DVD vers la seconde moitié des années 1990 a donné aux traductologues l'outil parfait pour mener des études comparatives, permettant un accès facile et instantané à des films dans leur version originale ainsi que doublée et sous-titrée. Citons en exemple l'étude que fait Jean-Pierre Mailhac des différentes versions anglaises du film français Gazon maudit, dont la conclusion est que, dans ce cas, le doublage assure une reproduction bien plus fidèle des dialogues originaux, alors que le sous-titrage n'en donne qu'une impression (Mailhac, 2000).

Toujours dans ce domaine, on observe le phénomène important du fansubbing, par lequel les amateurs de productions audiovisuelles non commercialisées dans leur marché (principalement des séries d'animation asiatiques) s'improvisent traducteurs et se chargent du sous-titrage, s'aidant mutuellement au sein d'une communauté en ligne étonnamment bien structurée. Un phénomène similaire, le $R O M$ hacking, ou translation hacking, s'observe également: des passionnés de jeux vidéo extraient le texte qui apparaît à l'écran et les dialogues des personnages pour les traduire puis les réintégrer au jeu, dans le but de distribuer une version artisanale, adaptée pour une région où le jeu n'est pas commercialisé et ne possède pas, par conséquent, de traduction "officielle». Les normes plus ou moins formelles propres au fansubbing, qui permettent de transmettre des informations aux spectateurs différemment qu'avec les sous-titres traditionnels et de les intégrer davantage au contenu visuel du cadre, ont piqué la curiosité de quelques chercheurs :

In one respect, fansubbing resorts to semiotic meaningmaking resources which are typically under-exploited in commercial subtitling - such as the choice of colours and fonts or the more intrusive insertion of interpretationframing notes on the part of the fansubber. Insofar as these resources are deployed concurrently, fansubbed films constitute complex gestalts of stimuli aiming to transmit 
more information through more sensory dimensions of the acoustic and visual channels. (Pérez González, 2007, p. 273-274)

Il est facile d'établir un lien entre le fansubbing et le phénomène de l'externalisation ouverte (crowdsourcing), qui permet à une organisation de faire appel à la communauté d'internautes, notamment pour traduire, puis réviser bénévolement le contenu textuel et audiovisuel qu'elle publie en ligne.

Un autre domaine de la traduction audiovisuelle, déjà un peu plus discret en traductologie, est celui de la traduction (et de l'adaptation) théâtrale et du surtitrage, que l'on rencontre principalement à l'opéra et dans les comédies musicales. Bien que le traducteur de pièces de théâtre ne doive produire qu'un texte écrit dans la langue cible, il s'agit tout de même de traduction audiovisuelle, car les paroles traduites sortiront de la bouche de personnages dans un environnement donné pendant une performance scénique (à l'exception de rares cas où le texte est traduit uniquement à des fins d'études). La traduction de dialogues soulève les questions de l'oralité du texte cible, du respect de la trame narrative (histoire personnelle du personnage, ton, niveau de vocabulaire, etc.) et de la performability, notion qui renvoie aux efforts du traducteur pour rédiger des répliques qui permettront aux acteurs d'être à leur meilleur et de maximiser les effets de leur performance (nous reviendrons sur cette notion clé plus loin). La traduction de pièces de théâtre et le surtitrage sous-tendent en outre un aspect sociologique de la traduction qui mériterait aussi qu'on s'y intéresse davantage en traductologie, celui de l'implication active du traducteur dans la production collective d'une œuvre. $\mathrm{Au}$ lieu de simplement remettre un texte cible, le traducteur est souvent appelé à assister aux répétitions pour voir sa version prendre forme et éventuellement la modifier en discutant avec les acteurs. Dans le même ordre d'idées, le traducteur de surtitres est souvent responsable de la projection du texte au-dessus des acteurs sur scène. Il doit alors assister lui aussi aux répétitions, non seulement pour connaître le texte sur le bout des doigts, mais également pour mieux connaître l'interprétation des acteurs et le rythme de leur jeu. C'est ainsi que le traducteur développera une sensibilité encore plus profonde au texte et une complicité avec les acteurs, ce qui lui permettra de mieux prévoir et gérer les variations 
dans le jeu, les changements de débit et de longueur des silences, de même que les erreurs et les trous de mémoire (des logiciels de surtitrage permettent la modification ou l'inversion des surtitres en temps réel, pendant la représentation).

Enfin, il existe un autre volet à la traduction audiovisuelle qui, comme nous l'avons mentionné en introduction, demeure très discret en traductologie, même s'il pose des problèmes très particuliers aux traducteurs et constitue une pratique plus répandue qu'on pourrait le croire, celui de la traduction de scénarios. C'est pourquoi nous proposons de placer sous les projecteurs cette forme de traduction spécialisée qui combine des éléments des autres formes de traduction audiovisuelle que nous venons de décrire, des contraintes techniques et temporelles, et des défis liés au respect de l'oralité et du contexte communicationnel.

\section{Le scénario comme outil de travail et la traduction scénaristique}

S'il est pertinent d'inclure la traduction scénaristique à la réflexion traductologique à propos des documents audiovisuels, c'est notamment parce que ses enjeux et défis sont de taille. Le traducteur n'a peut-être pas à composer avec les contraintes habituellement associées à l'audiovisuel, comme le temps de lecture pour le sous-titrage ou la correspondance du mouvement des lèvres pour le doublage, mais il doit maîtriser le langage technique de la scénarisation, les principes de la rédaction de dialogues et même, dans certains cas, le fonctionnement des structures narratives. Tout d'abord, rappelons que le scénario est un document écrit structuré et complet, qui présente ce qui sera vu et entendu pendant la présentation du produit final, sous toutes les formes qui soient (contenu visuel, texte apparaissant à l'écran, dialogues, effets sonores, musique). La scénarisation est par le fait même un hybride entre la création littéraire et la rédaction technique; elle respecte un ensemble de normes quant au fond et, surtout, quant à la forme. C'est le plan d'un récit scène par scène qui prendra forme pendant le tournage et sera achevé au montage. Sa présentation peut varier selon le type de document audiovisuel ou l'équipe de production, mais elle adopte généralement la forme suivante: 
INT. SALLE DE CLASSE - JOUR

Un PROFESSEUR entre dans une salle de classe remplie de jeunes élèves, alors que la cloche sonne.

\section{PROFESSEUR}

\section{Ouvrez vos livres à la page 32.}

Les élèves rouspètent en cœur.

Figure 1. Modèle de scénario traditionnel

C'est le modèle utilisé pour les œuvres de fiction et pour la plupart des autres productions, mais il existe aussi un modèle, par exemple, dans lequel chaque scène est divisée en deux colonnes afin de distinguer le contenu visuel et sonore.

\begin{tabular}{|l|l|}
\hline VIDÉO & AUDIO \\
\hline $\begin{array}{l}\text { Une salle de classe remplie } \\
\text { d'élèves. }\end{array}$ & E. s.: Sonnerie de cloche. \\
$\begin{array}{l}\text { Un PROFESSEUR entre dans } \\
\text { la pièce. }\end{array}$ & $\begin{array}{l}\text { PROFESSEUR: } \\
\text { Ouvrez vos livres à la page 32. }\end{array}$ \\
$\begin{array}{l}\text { Les élèves s'agitent derrière leur } \\
\text { bureau. }\end{array}$ & $\begin{array}{l}\text { E. s.: Murmures de protestation } \\
\text { de tous les élèves. }\end{array}$ \\
\hline
\end{tabular}

Figure 2. Modèle de scénario traditionnel avec distinction du contenu visuel et sonore

Pour le traducteur, la partie descriptive du scénario ne pose habituellement aucun problème. Il suffit de conserver le même style direct, avec des phrases courtes, simples, toujours au présent. En cas de doute, ou s'il doit modifier une scène, il devra ne jamais oublier qu'il ne peut décrire que ce qui apparaît ou est entendu. En d'autres mots, un personnage n'est pas simplement nerveux, il fait plutôt les cent pas ou se ronge les ongles. La traduction de dialogues s'avère quant à elle bien plus complexe. Outre le sens des paroles, le traducteur doit prendre en considération le contexte et l'identité du personnage, sans oublier que ce texte sera lu et joué par un comédien. En plus d'être agréable à l'oreille, ce texte devra être facilement perceptible et compréhensible à la première écoute, tout en permettant au comédien d'être à son meilleur. Il y a clairement un rapprochement à faire entre cet aspect de la 
traduction scénaristique et la notion théorique de performability en traduction théâtrale, définie comme "the ability to produce fluid texts which performers may utter without difficulty" (Espasa, 2000, p. 49). Il est clair que le traducteur ne peut ignorer cette responsabilité. Toutefois, l'idée même de performability est parfois critiquée, car elle pourrait laisser entendre que le dialogue traduit est nécessairement moins efficace que le dialogue original. Susan Bassnett, notamment, réfute cette notion qui a pourtant fait l'objet d'un bon nombre de publications en traductologie: "I have great problems with 'performability'. It seems to me a term that has no credibility, because it is resistant to any form of definition» (Bassnett, 1998, p. 95). Bref, le traducteur est appelé à considérer l'éventuelle performance du comédien, sans pour autant oublier qu'il doit d'abord et avant tout se concentrer sur l'aspect linguistique du travail.

Par ailleurs, le traducteur doit être conscient que le scénario est plus qu'un collage descriptif de scènes. D'après la professeure de cinéma Esther Pelletier, c'est surtout un «plan détaillé du récit qui prendra forme au tournage et sera parachevé au montage dans sa version définitive» $(1995$, p. 30) et qui ensuite "aura pour fonction de servir de plan de travail et de guide à l'équipe de production, au réalisateur et aux différentes équipes à la réalisation, aux comédiens, aux musiciens ainsi qu'à l'équipe du montage" (ibid.). Ainsi, plutôt que de fournir un document final qui sera lu et perçu tel quel par le spectateur, le scénariste, et par conséquent le traducteur de scénarios, doit produire ni plus ni moins qu'un outil de travail sur lequel reposeront les activités des producteurs, artistes et artisans de la production. Il faut être conscient que ce document ne cherche pas à être un produit final; il est au contraire susceptible d'évoluer pendant les différentes étapes de production. Il est donc possible d'établir un lien ici entre les scénarios et les pièces de théâtre, car, dans les deux cas, le traducteur «is still left with the task of transforming unrealized text $A$ into unrealized text $\mathrm{B} »($ Bassnett, 1991, p. 101). Le traducteur doit comprendre les implications techniques et artistiques de chaque élément du scénario, et pouvoir travailler avec les outils de référence que sont le découpage technique et le scénarimage (ou storyboard en anglais), une représentation illustrée du scénario qui peut s'apparenter à une bande dessinée. En effet, il arrive encore souvent que le traducteur se retrouve tout au bout de la chaîne et 
qu'il doive travailler avec ce type de document (notamment dans le cas de documentaires et de reportages, où le spectateur n'entend qu'une voix hors champ, comme celle d'un narrateur). Ainsi, il est pertinent de s'interroger sur les connaissances et les aptitudes que doit posséder le traducteur pour produire un document aux usages aussi variés. Dans un court chapitre qui constitue à notre connaissance un des très rares écrits traductologiques exclusivement consacrés à la traduction scénaristique, Patrick Cattrysse et Yves Gambier avancent que les scénarios devraient être traduits non pas par des scénaristes bilingues, mais bien par des traducteurs professionnels ayant été formés pour la rédaction cinématographique:

The transdisciplinary answer would be that one should start to acquire the professional knowledge and skills that are needed to do the job and then proceed to design a training programme that would help trainees learn the required knowledge and skills. (Cattrysse et Gambier, 2008, p. 55)

Bien entendu, si le doublage et le sous-titrage occupent une place prédominante au sein des recherches sur la traduction audiovisuelle, c'est notamment parce que ces types de traduction sont les plus fréquemment utilisés pour faire voyager les productions cinématographiques et télévisuelles d'une culture à l'autre. Il est cependant important de comprendre pourquoi d'autres types de traduction sont parfois préférés et, dans le cas qui nous intéresse, d'établir quelles sont les raisons de traduire un scénario. Cattrysse et Gambier donnent une première réponse, bien qu'elle soit très ancrée dans la réalité européenne. Ils expliquent que la traduction de scénarios est une pratique incontournable pour une industrie cinématographique dans laquelle les coproductions multinationales sont monnaie courante et qu'il s'agit par conséquent d'un élément clé pour intéresser les producteurs étrangers. Ainsi, un producteur italien peut recevoir une version italienne du scénario d'un film français en développement afin qu'il puisse mieux connaître le film pour lequel il est appelé à investir des sommes importantes. Dans ce cas, le scénario traduit ne doit pas seulement être rigoureusement précis quant au contenu du film, mais il doit également pouvoir séduire par une langue vive et agréable. Les dialogues doivent être à la fois naturels, fidèles à la volonté artistique de l'œuvre, 
drôles ou touchants si nécessaire, et surtout crédibles lorsqu'ils sont prononcés dans la bouche d'un personnage qui a sa propre personnalité, son histoire, son milieu social, etc. Une seconde raison pour traduire un scénario est liée à l'aspect pratique de ce document de travail utilisé par les différentes équipes créatives mentionnées plus haut. Dans un contexte de coproduction, ces équipes peuvent provenir de différents pays; elles ont toutes besoin de bien connaître le contenu du film pour être inspirées et pour saisir la nature et l'ampleur de leur tâche. Si des créateurs d'effets spéciaux allemands sont engagés pour travailler sur un film français, ils doivent connaître l'histoire et le propos $\mathrm{du}$ film dans son ensemble, et non uniquement le contenu des scènes auxquelles ils contribueront. D'un point de vue théorique, il est facile d'établir un lien avec cet aspect de la traduction scénaristique et la théorie du Skopos en traductologie développée par Katharina Reiss et Hans Vermeer (1984), dans laquelle la traduction est considérée comme un acte de communication orienté autour de l'objectif du texte cible: «one must translate [...] in accordance with some principle respecting the target text. The theory does not state what the principle is: this must be decided separately in each specific case» (Vermeer, 2000, p. 228). On fait traduire un scénario pour une raison bien précise; il sera destiné à une personne en particulier qui en fera une lecture bien spécifique selon ses besoins. Christiane Nord insiste d'ailleurs sur l'importance de ce lecteur cible auquel la traduction est destinée: «the receiver, or rather the addressee, is the main factor determining the target-text Skopos» (1997, p. 29). Le traducteur ne doit donc jamais oublier que la traduction d'un scénario peut à la fois aider des techniciens à planifier un calendrier de travail de plusieurs semaines, guider et inspirer des décorateurs ou des concepteurs de costumes, ou encore frapper l'imaginaire d'un producteur pour lui donner le goût d'investir dans un projet.

Cela dit, bien que ce soient les grandes productions cinématographiques qui volent la vedette dans la majorité des études traductologiques sur l'audiovisuel, il existe beaucoup d'autres types de vidéos sur lesquels des traducteurs sont appelés à travailler. On peut penser aux vidéos d'entreprise dans lesquels le président d'une multinationale souhaite la bienvenue aux nouveaux employés et présente le fonctionnement de son organisation, aux didacticiels animés, aux vidéos de formation, 
etc. Il faut aussi ajouter à cette liste la publicité, alors que les campagnes se déclinent de plus en plus sur les écrans en tous genres, et où la traduction scénaristique prend un aspect peut-être plus concret qu'avec les scénarios qui ne sont traduits qu'à des fins informatives. En effet, avec les doubles versions notamment, le traducteur rédige un scénario qui ne sera pas que lu, mais qui est destiné à être joué et enregistré au même titre que le scénario original. Il s'agit d'une pratique courante qui demeure pratiquement inexplorée en traductologie, même si elle place le traducteur au cœur d'une opération de production et l'incite parfois à outrepasser son rôle d'expert langagier pour devenir un véritable conseiller culturel.

\section{La double version, ou le scénario comme matière première du traducteur}

Produire une double version (aussi nommée double tournage, et double shoot ou double shooting en anglais) signifie recréer le message audiovisuel pour la culture cible à partir de l'adaptation $\mathrm{du}$ scénario du produit original. Bref, au lieu de doubler ou de sous-titrer le document audiovisuel original, il s'agit d'en traduire et d'en adapter le scénario pour ensuite tourner une version propre au nouveau public cible, avec des acteurs qui en maîtrisent bien sûr la langue cible et qui appartiennent à la même culture. Cette opération de traduction, résolument ethnocentrique, correspond à la définition de l'adaptation que propose Julie Sanders: "[an] attempt to make text 'relevant' or easily comprehensible to new audiences and readerships via the processes of proximation and updating» (2006, p. 19). Lorsque cela est possible, cette version réécrite avec le nouveau public cible en tête est tournée immédiatement après la version originale, afin de profiter de la présence de l'équipe technique, du studio et des décors déjà installés, et par le fait même de minimiser les coûts de production. Il faut par ailleurs distinguer la double version du remake, un emprunt culturel très ethnocentrique assimilable à l'appropriation, où une œuvre est entièrement recréée pour une autre culture. Comme d'innombrables exemples hollywoodiens en font la démonstration, les remakes sont par nature des œuvres postérieures à l'original, qui peuvent n'avoir en commun avec ce dernier que quelques éléments souvent davantage liés au fond qu'à la forme du produit. Les doubles versions s'inscrivent dans 
une stratégie communicationnelle plus globale, dans laquelle les scénaristes savent souvent d'emblée que leur travail sera éventuellement traduit afin de tourner, simultanément ou non, des versions en différentes langues (ce qui influence bien sûr la rédaction du scénario dit original).

Le processus de production d'une double version demeure plus complexe que le sous-titrage et même que le doublage, et, avant d'opter pour cette solution, le client doit sérieusement en considérer les avantages et les inconvénients. Le traducteur expérimenté est d'ailleurs souvent bien placé pour conseiller son client en tenant compte de divers facteurs, dont le contexte de communication, le budget disponible, le calendrier de production souhaité, le public visé et les médias retenus. Les inconvénients des doubles versions sont manifestes. Le processus est long, puisqu'il faut adapter le scénario, suivre les étapes normales de production, dont la distribution, pour trouver des acteurs de la culture cible qui, normalement, ressemblent aux acteurs de l'original, effectuer le montage et la postproduction, etc. Par le fait même, le deuxième inconvénient des doubles versions est leur coût imposant: il faut payer les acteurs, le réalisateur, le temps de studio et les techniciens. Tout cela est bien plus cher que l'embauche d'un traducteur pour sous-titrer un document ou que celle d'une équipe pour effectuer un doublage de qualité professionnelle (sans oublier la location du studio d'enregistrement). Par contre, et c'est là le premier avantage des doubles versions, ces coûts importants peuvent être amoindris par des économies d'échelle lorsque le tournage de l'adaptation se fait simultanément à celui de l'original. Toutefois, c'est l'adaptabilité maximale qui rend cette pratique si intéressante dans un contexte de communication publicitaire. Les contraintes $\mathrm{du}$ sous-titrage ou du doublage, alors que la matière première du traducteur est non plus le texte, mais une image déterminée et absolument inchangeable, sont bien connues. Dans le cas de la double version, le traducteur est libéré de ces contraintes. Il a le champ libre pour maximiser la qualité de sa traduction, quitte à utiliser des phrases un peu plus longues ou à en changer complètement la structure lorsque c'est possible. Cela lui accorde une plus grande marge de manœuvre pour trouver des solutions satisfaisantes et efficaces aux problèmes traductionnels auxquels il est confronté, ce qui est particulièrement utile avec les scènes comprenant des références culturelles ou teintées d'humour. En 
conservant toujours l'objectif du client et les fonctions de la vidéo en tête (divertir, former, séduire, vendre...), le traducteur peut ainsi choisir les solutions qui lui semblent les plus appropriées. Dans le même ordre d'idées, une double version réussie sera normalement perçue et comprise plus aisément par le spectateur, à qui l'on s'adresse directement, dans sa langue et avec son accent, tandis que dans bien des cas, le doublage, et particulièrement le sous-titrage, peuvent créer une certaine distance. Finalement, avec la double version, le traducteur peut devenir un véritable agent culturel. À ce titre, il bénéficie d'une plus grande autonomie et peut même être appelé à participer aux différentes étapes de production (préparation, distribution, auditions, tournage, etc.), toujours en tant qu'expert de la culture cible. À l'instar du traducteur de pièces de théâtre qui discute avec le metteur en scène et assiste aux répétitions, le traducteur de scénarios peut être présent sur le plateau de tournage pour orienter le jeu des acteurs qui donnent vie aux dialogues et aux narrations qu'il a écrits. Par ailleurs, en l'absence d'un producteur de la culture cible, le traducteur peut devoir mettre son poing sur la table pour défendre sa version et empêcher, par exemple, que l'équipe de tournage de l'original passe la majeure partie du temps de tournage à peaufiner sa version et qu'il ne reste ensuite que très peu de temps pour tourner la version adaptée, qui sera bouclée en quelques prises. De plus, en assistant à l'enregistrement de l'adaptation en studio, en compagnie des techniciens et des artistes, le traducteur acquiert une expérience pratique du jeu et de la rédaction scénaristique qui lui sera très utile, d'autant plus que ces notions n'ont fort probablement pas été abordées en profondeur durant sa formation universitaire.

\section{Traduction scénaristique et double version en publicité}

S'il existe un domaine dans lequel la traduction scénaristique en vue de produire des doubles versions offre des avantages stratégiques évidents par rapport aux autres formes de traduction audiovisuelle, c'est bien celui de la publicité. En cette époque de mondialisation et de prolifération des technologies de l'information et de la communication, les stratégies de marketing utilisées pour vendre et séduire ont connu des changements importants, particulièrement dans les marchés secondaires. Aujourd'hui, la tendance est de traduire et d'adapter un seul message conçu pour fonctionner dans de nombreuses cultures 
au lieu de créer des campagnes différentes pour chaque marché distinct. En plus de permettre d'évidentes économies d'échelle, cette façon de faire assure un contrôle très serré de l'identité de la marque et facilite grandement le façonnement de la perception d'une identité et d'une image cohérentes chez des publics donnés. Toutefois, une campagne pensée et façonnée à New York ou à Paris peut-elle rejoindre et séduire les consommateurs de partout? Dans un article sur l'adaptation en traduction, nous avons déjà réfléchi aux mécanismes de la réception des publicités traduites dans la culture cible:

the advertisement must not only reach its intended target, but also create the belief that the ad speaks directly to individual viewers. [...] In fact, any indication that the ad has been created in a distinct culture might jeopardize the viewer's ability and "willingness" to feel concerned by the message and to comprehend its content. (Vandal-Sirois et Bastin, 2012, p. 31-32)

Grâce aux avantages énumérés précédemment, la double version permet de créer un certain rapport de proximité entre l'annonceur et la cible, ce qui facilite grandement le phénomène clé en publicité qu'est ce processus d'identification. De plus, l'implication active du traducteur, non seulement dans l'opération de transfert linguistique, mais également dans les autres étapes de la préproduction et de la postproduction, peut s'avérer très bénéfique pour le client, surtout s'il connaît peu la culture cible à laquelle il souhaite s'adresser. L'utilisation de porte-parole célèbres est un bon exemple. L'annonceur peut souhaiter utiliser une vedette dans les messages présentés à l'étranger. Dans un tel cas, cette vedette sera doublée dans la langue cible ou, dans des cas plus rares, on lui demandera de prononcer le texte traduit vers la langue cible... avec des résultats souvent discutables! La production d'une double version est une autre possibilité: la célébrité de l'original sera alors remplacée par une célébrité locale dont les attributs et la crédibilité s'avèrent comparables. Encore une fois, le traducteur pourra ici servir d'agent culturel et suggérer des candidats au client étranger, qui aurait bien de la difficulté à faire un choix éclairé sans une personne-ressource intégrée à la culture cible. Par ailleurs, comme la communication publicitaire est très réglementée par différentes agences gouvernementales, 
qui varient d'un pays à l'autre ${ }^{1}$, le traducteur peut être parmi les premiers intervenants à repérer dans un scénario des éléments susceptibles d'être interdits dans sa culture (les normes relatives à la publicité de boissons alcoolisées et de produits pharmaceutiques de même que celles relatives aux publicités destinées aux enfants sont particulièrement pointilleuses). Bien que les avocats de la filiale ou de l'agence de l'annonceur située dans la culture cible analyseront vraisemblablement le message final, le traducteur qui connaît les normes en vigueur pourra faire gagner beaucoup de temps à son client en repérant les problèmes éventuels et en proposant des solutions.

\subsection{Exemple de double version en publicité télévisée}

Afin de mieux saisir la portée du travail du traducteur et des autres professionnels de la communication impliqués dans la création d'une double version, nous proposons d'examiner une publicité télévisée de 30 secondes de l'entreprise de télécommunications Telus, créée par l'agence Taxi et diffusée à l'automne 2012. Le concept est simple: il s'agit d'informer les jeunes consommateurs que l'entreprise élimine les frais de mise en service pour les nouveaux clients, en rappelant que payer de tels frais est exaspérant. Dans les versions anglaise et française, un jeune homme dont les vêtements et le sac en bandoulière peuvent évoquer à la fois un étudiant et un jeune professionnel s'adresse directement à la caméra en disant que payer des frais de mise en service est encore plus énervant que son colocataire excentrique.

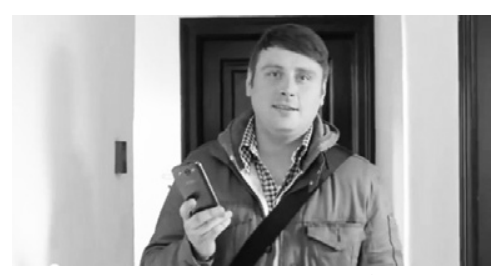

Image 1. Version anglaise

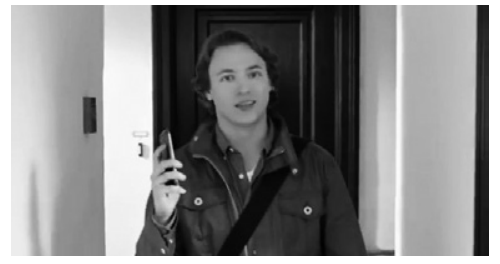

Image 2. Version française

1. Au Canada, les critères d'acceptabilité des publicités sont présentés dans le Code canadien des normes de la publicité de l'organisme d'autoréglementation des Normes canadiennes de la publicité (http://www.adstandards.com/fr/ Standards/theCode.aspx). 
Comme nous le voyons dans les images 1 et 2, les deux versions ont été tournées dans le même décor, avec des acteurs au style plutôt semblable, choisis spécifiquement pour chaque public. Dans cette première partie du message, le propos est le même, et le monologue est traduit presque littéralement: «I just got a new phone. Amazing! But paying to activate it... even more annoying that my new roommate» devient «J'adore mon nouveau téléphone. Mais payer pour des frais de mise en service, c'est encore plus énervant que mon coloc.» La mise en situation est pratiquement identique et, dans les deux cas, le ton est naturel et le vocabulaire utilisé est crédible avec le personnage auquel le public ciblé est censé s'identifier d'emblée (notamment avec l'expression abrégée coloc, pour colocataire).

Pour la conception d'une double version réussie, c'est l'ensemble du produit fini que le traducteur et les autres parties impliquées doivent considérer, et non uniquement la traduction du scénario elle-même. Le présent cas en est un bon exemple. En arrière-plan de l'image 3, un panneau Exit est bien visible, indiquant que la publicité a probablement été tournée dans un environnement anglophone. Le plan de caméra plus serré de la version française (image 4) supprime cet élément qui risquait de rompre la cohérence du message et de nuire au phénomène crucial d'identification que la double version facilite... à moins que ce genre de détail ne soit négligé.

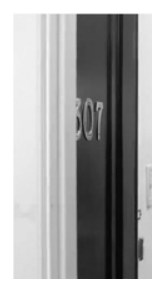

Image 3. Version anglaise

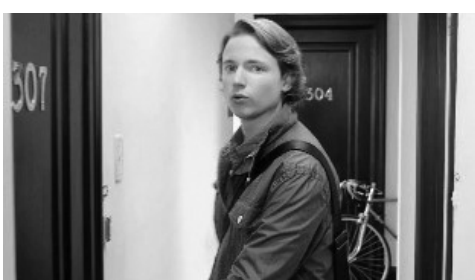

Image 4. Version française

Bien entendu, il n'incombe pas au traducteur de faire le travail du cadreur ou du réalisateur, mais il doit mettre son intuition et son bagage culturel au profit du message final, et ne pas hésiter à signaler ce genre de situation aux membres des équipes techniques et de création, surtout lors du tournage d'une version dans une langue qu'ils ne comprennent pas et qui s'adresse à une culture qui leur est étrangère. 
Ensuite, dans les deux versions, le personnage illustre son propos en ouvrant la porte de son appartement pour montrer que son colocataire est effectivement très énervant. Ici, Telus et son agence ont choisi d'engager une personnalité connue pour incarner le colocataire, stratégie très fréquente en publicité pour profiter de la notoriété d'une célébrité et attirer l'attention du public ciblé. Bien entendu, l'image de cette personnalité doit être cohérente avec le concept, et c'est pourquoi les concepteurs sont allés chercher le flamboyant entraîneur Richard Simmons. Dans une chorégraphie qui évoque ses vidéos d'exercice, Simmons annonce à son malheureux colocataire qu'il a utilisé tout le détergent à lessive pour laver ses fameux vêtements pastel.

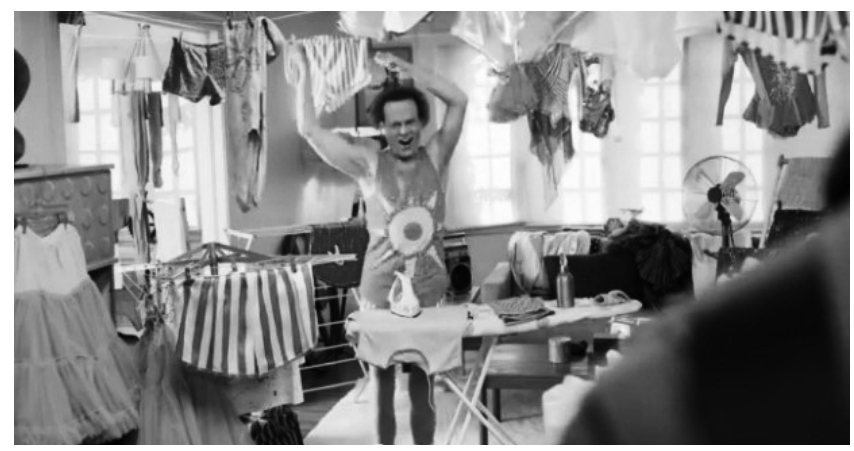

Image 5. Version anglaise - Richard Simmons

Pour la version diffusée au Québec, le personnage principal entre dans un appartement complètement différent où le colocataire, tout aussi excentrique, est joué par l'humoriste québécois André Sauvé. Comme Simmons, unilingue anglophone, est peu connu au Québec, ses vidéos d'exercice n'ayant jamais été doublées ni commercialisées en français, il était logique d'opter pour un personnage loufoque qui serait plus facilement reconnaissable par le public québécois. Comme ceétait le cas avec Simmons, la mise en scène avec le personnage interprété par Sauvé respecte à la fois le propos du message et l'univers qui a fait la renommée de l'interprète (image 6). Plus précisément, Sauvé manipule de curieux appareils, à la recherche de «mauvaises énergies» qu'il attribue à son colocataire. 


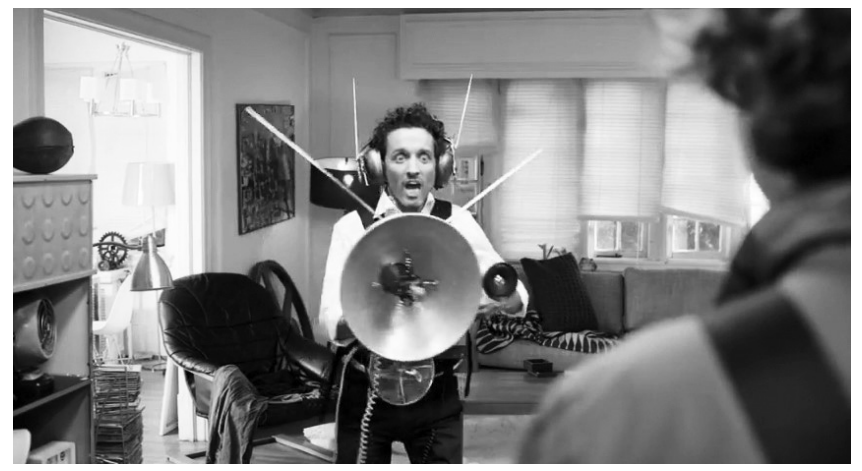

Image 6. Version française - André Sauvé

Par ailleurs, cette adaptation a également entraîné un changement au niveau de la trame musicale qui accompagne les images: alors que la version anglaise contient une pièce disco similaire à celles qui sont caractéristiques des vidéos de Simmons, la version française présente une composition du groupe québécois Bravofunken, toujours dans le but de «rapprocher» la marque de ses cibles. Finalement, les deux versions se terminent par une séquence de phrases écrites qui rappellent le propos du message ("We hear you. We got rid of activation fees.»/«Nous vous avons entendus. Nous éliminons les frais de mise en service.»), puis par la signature de l'entreprise et une adresse URL qui mène vers la page Web anglaise ou française, selon le cas. Dans le cas de la signature, le traducteur doit bien entendu effectuer un travail de recherche avant de l'adapter, car il en existe déjà une traduction officielle dans la majorité des cas (images 7 et 8).

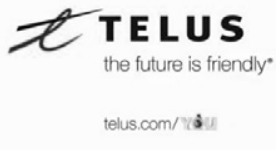

Image 7. Version anglaise

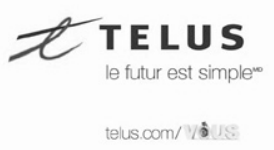

Image 8. Version française

Cet exemple illustre bien les différentes facettes de la traduction scénaristique ainsi que la nature hétérogène du travail du traducteur œuvrant dans ce domaine. Il n'est pas sans rappeler les propos de Jean Delisle, selon qui « la cloison séparant la création, 
l'interprétation, la recréation, la traduction et l'adaptation n'est pas si étanche qu'on a tendance à le croire» (1980, p. 81).

\section{Conclusion}

Contrairement à ce que laisse croire la très grande visibilité du doublage et du sous-titrage, la traduction de scénarios est une forme de traduction audiovisuelle répandue, en Europe comme en Amérique du Nord. Il s'agit d'une activité complexe, de nature variée, qui peut comprendre des éléments de traduction juridique et technique ou pousser le traducteur à adopter une approche plus libre ou créatrice, toujours selon la fonction du document audiovisuel et le contexte communicationnel. D'une certaine façon, le traducteur est libéré des contraintes et difficultés habituellement liées à la traduction audiovisuelle, mais il doit par contre considérer un tout autre ensemble de particularités traductionnelles. Il est d'ailleurs étonnant que le sujet n'ait pas été davantage exploré en traductologie, surtout en cette époque de prolifération des moyens de communication, où la traduction audiovisuelle devient plus importante que jamais. Par ailleurs, les responsabilités élargies du traducteur lors de la production d'une double version, qui vont bien au-delà des préoccupations exclusivement linguistiques, et son statut d'agent culturel auprès de son client étranger correspondent parfaitement aux plaidoyers de Lawrence Venuti et de Douglas Robinson, notamment, quant au rôle que peut jouer le traducteur dans le processus de production d'un texte. Dans le même ordre d'idées, le traducteur de scénarios qui participe activement à la production de la vidéo cible, rencontre les comédiens et prend sa place sur le plateau de tournage afin d'assurer le meilleur produit possible est à l'image de la perception du métier de traducteur présentée, entre autres, dans Enlarging Translation, Empowering Translators de Maria Tymoczko (2007), qui confère au traducteur une autonomie accrue dans ses prises de décision. Évidemment, cette autonomie correspond à de plus grandes responsabilités, qui ne sont possibles que lorsque le traducteur a, d'une certaine façon, gagné la confiance de ses collègues et clients. Pour y arriver, et pour que les scénarios soient traduits non pas par un scénariste qui s'improvise traducteur, mais plutôt par un traducteur expérimenté en rédaction scénaristique, l'approche multidisciplinaire que préconisent Cattrysse et Gambier pour la formation des traducteurs est un bon point de départ. Toutefois, il 
faut également favoriser l'intégration active des traducteurs dans les différentes sphères d'activité où ils sont appelés à travailler, afin de mieux saisir la nature de leur contribution auprès de leurs collègues non traducteurs et, ultimement, de valoriser leur rôle jusqu'à ce qu'il atteigne son plein potentiel. C'est par une telle approche synergique que la petite histoire d'un scénario traduit pour inspirer des artistes, séduire des investisseurs ou guider la production d'une vidéo aussi drôle, touchante, informative, bref, efficace que le document original connaîtra un bappy-ending.

\section{Références}

Bassnett, Susan (1991). "Translating for the Theatre: The Case Against Performability». TTR, 4, 1, p. 99-111.

Bassnett, Susan (1998). "Still Trapped in the Labyrinth: Further Reflections on Translation and Theatre». In S. Bassnett et A. Lefevere, dir. Constructing Cultures: Essays in Literary Translation. Clevedon, Multilingual Matters, p. 90-108.

Cattrysse, Patrick et Yves Gambier (2008). "Screenwriting and Translating Screenplays». In J. Díaz Cintas, dir. The Didactics of Audiovisual Translation. Amsterdam/Philadelphie, John Benjamins, p. 39-55.

Delisle, Jean (1980). L'analyse du discours comme méthode de traduction. Initiation à la traduction française de textes pragmatiques anglais: théorie et pratique. Ottawa, Éditions de l'Université d'Ottawa.

Díaz Cintas, Jorge, dir. (2008). The Didactics of Audiovisual Translation. Amsterdam/Philadelphie, John Benjamins.

Díaz Cintas, Jorge, dir. (2009). New Trends in Audiovisual Translation. Amsterdam/Philadelphie, John Benjamins.

Espasa, Eva (2000). «Performability in Translation: Speakability? Playability? Or Just Saleability?» In C.-A. Upton, dir. Moving Target, Theatre Translation and Cultural Relocation. Manchester, St. Jerome, p. 49-62.

Mailhac, Jean-Pierre (2000). «Subtitling and Dubbing, For Better or Worse? The English Video Versions of Gazon maudit ». In M. SalamaCarr, dir. On Translating French Literature and Film II. Amsterdam et Atlanta, Rodopi, p. 129-154.

Nord, Christiane (1997). Translation as a Purposeful Activity: Functionalist Approaches Explained. Manchester, St. Jerome.

O’Hagan, Minako (2009). «Putting Pleasure First: Localizing Japanese Video Games ». TTR, 22, 1, p. 147-165. 
Orero, Pilar, dir. (2004). Topics in Audiovisual Translation. Amsterdam/ Philadelphie, John Benjamins.

Pedersen, Jan (2011). Subtitling Norms for Television. Amsterdam/ Philadephia, John Benjamins.

Pelletier, Esther (1995). Écrire pour le cinéma: le scénario et l'industrie du cinéma québécois. Québec, Nuit Blanche Éditeur.

Pérez González, Luis (2007). «Fansubbing Anime: Insights into the 'Butterfly Effect' of Globalisation on Audiovisual Translation». Perspectives: Studies in Translatology, 14, 4, p. 260-277.

Reiss, Katharina et Hans J. Vermeer (1984). Grundlegung einer allgemeinen Translationstheorie. Tübingen, Niemeyer.

Sanders, Julie (2006). Adaptation and Appropriation. Londres et New York, Routledge.

Tymoczko, Maria (2007). Enlarging Translation, Empowering Translators. Manchester, St. Jerome.

Vandal-Sirois, Hugo et Georges L. Bastin (2012). «Adaptation and Appropriation: Is There a Limit?» In L. Raw, dir. Translation, Adaptation and Transformation. New York/Londres, Continuum, p. 21-41.

Vermeer, Hans J. (2000). «Skopos and Commission in Translational Action ». Trans. Andrew Chesterman. In L. Venuti, ed. The Translation Studies Reader. Londres et New York, Routledge, p. 221-232.

Hugo Vandal-Sirois

Université de Montréal Département de linguistique et de traduction

Pavillon Lionel-Groulx 3150, rue Jean-Brillant, bur. C-9006

Montréal (Québec) H3T 1N8 CANADA hugo.vandal-sirois@umontreal.ca 